\title{
FOUR BRIEF EXAMPLES CONCERNING POLYNOMIALS ON CERTAIN BANACH SPACES
}

\author{
ROBERT A. BONIC
}

Let $E$ denote one of the spaces $l^{p}(1 \leq p<\infty)$ or $c_{0}$, and $\left\{e_{1}, e_{2}, \cdots\right\}$ be the standard basis in $E$. An element $x$ in $E$ will be written as $x=\Sigma_{n} x_{n} e_{n}$. The following examples are perhaps justified by the fact that their proofs are shorter than their statements.

Example A. Suppose $E$ is real, and

$$
\phi(t)=3 t^{2}-2 t^{3}(t \text { real }), \quad \phi_{n}(t)=\phi\left(\alpha_{n} t\right) / 2^{n-1},
$$

where $\alpha_{n}=2^{n / 4}$. Then the mapping $A(x)=\Sigma_{n} \phi_{n}\left(x_{n}\right)$ is a continuous realvalued polynomial of degree 3 , and the image of the critical points contains $[0,2]$.

Proof. Any $x$ of the form $x=\Sigma_{n} \varepsilon_{n} \alpha_{n}^{-1} e_{n}$, where $\varepsilon_{n}$ is 0 or 1 , is a critical point of $A$, and $A(x)=\Sigma_{n} \varepsilon_{n} / 2^{n-1}$.

Example $A$ is based on examples of Kupka [2] and Bonic [1], and the remark "bien sur" of Douady [Baton Rouge, April 1967].

Example B. Suppose $E$ is complex, and

$$
\begin{aligned}
\varphi(z) & =a z^{2}+b z^{3}+c z^{4}+d z^{5} \\
\psi_{n}(z) & =\phi\left(\beta_{n} z\right) / 2^{n-1}, \\
4 a & =4 i+4, \quad 4 b=-5 i+5, \quad \beta_{n}=2^{n / 6} \\
4 c & =-2 i-2, \quad 4 d=3 i-3, \quad
\end{aligned}
$$

where $z$ is complex. Then the mapping

$$
B(x)=\Sigma_{n} \psi_{n}\left(x_{2 n-1}\right)+\Sigma_{n} \psi_{n}\left(x_{2 n}\right)
$$

is a continuous complex-valued polynomial of degree 5, and the image of the critical points contains $[0,2] \times[0,2]$.

Proof. Any $x$ of the form

$$
x=\Sigma_{n} \varepsilon_{n} \beta_{n}^{-1} e_{2 n-1}-\Sigma_{n} \delta_{n} \beta_{n}^{-1} e_{2 n},
$$

Communicated by R. S. Palais, March 18, 1968. Research supported in part by NSF Grant GP-7026. 
where $\varepsilon_{n}$ and $\delta_{n}$ are 0 or 1 , is a critical point of $B$, and $B(x)=\Sigma_{n} \varepsilon_{n} / 2^{n-1}$ $+i \Sigma_{n} \delta_{n} / 2^{n-1}$.

Example C. Suppose $E$ is real or complex. Then the mapping $C(x)$ $=\Sigma_{n} x_{n}^{2} e_{n}$ is a continuous, but not completely continuous, polynomial of degree 2 from $E$ into $E$, and each derivative of the polynomial is completely continuous.

Proof. $C$ is clearly continuous but cannot be completely continuous since $C e_{n}=e_{n}$ for all $n$. Since $D C(x) h=\Sigma_{n} 2 x_{n} h_{n}$ and $x_{n} \rightarrow 0$, we have that $D C(x)$ is completely continuous.

Example $\mathrm{C}$ answers a question asked the author by $\mathrm{A}$. Tromba who pointed out that it solves negatively a problem posed in Vainberg [4, p. 51].

Example D. Suppose $E$ is real $c_{0}$. Then the mapping $D(x)=\Sigma_{n}\left(x_{n}+x_{n}^{3}\right) e_{n}$ is a continuous polynomial of degree three from $c_{0}$ into $c_{0}$, and is a proper map (the inverse image of a compact set is compact). Moreover, $D$ has the form $D=I+D_{0}$, where $D_{0}$ is not completely continuous, but each derivative of $D_{0}$ is completely continuous.

Proof. Letting $\phi$ denote the inverse of the mapping $\psi(t)=t+t^{3}$ we have that $D^{-1}(x)=\Sigma_{n} \phi\left(x_{n}\right) e_{n}$ is a continuous map of $c_{0}$ into $c_{0}$ and hence that $D$ is proper. The facts about $D_{0}$ follow exactly as in Example $C$.

Example $\mathrm{D}$ is of some interest in degree theory since the usual LeraySchauder degree is given for maps of the form $I+F$, where $F$ is a completely continuous mapping. In [3] Tromba develops a degree theory for smooth proper maps of the form $I+G$ only assuming that each $D G(x)$ is completely continuous. Therefore this example gives an instance, where the latter but not the former degre is defined.

\section{Bibliography}

[1] R. A. Bonic, A note on Sard's theorem in Banach spaces, Proc. Amer. Math. Soc. 17 (1966) 1218.

[2] I. Kupka, Counterexample to the Morse-Sard theorem in the case of infinite dimensions, Proc. Amer. Math. Soc. 16 (1965) 954-957.

[3] A. J. Tromba, Degree theory on Banach manifolds, Princeton University Thesis, 1968.

[4] M. M. Vainberg, Variational methods for the study of nonlinear operators, HoldenDay, San Francisco, 1964.

NORTHEASTERN UNIVERSITY 